Binder/Glos/Riepe (Hrsg.)

Handbuch Bankenaufsichtsrecht 



\section{Handbuch Bankenaufsichtsrecht}

2., neu bearbeitete Auflage

herausgegeben von

Professor Dr. Jens-Hinrich Binder, LL.M. (London)

Rechtsanwalt Dr. Alexander Glos

Professor Dr. Jan Riepe

bearbeitet von

Dr. Dorothee Amann, RAin Dr. Denise Bauer-Weiler,

Dr. Frank Beekmann, RA Dr. Markus Benzing, LL.M. (Nottingham,

Trent University), RA Dr. Marc Benzler, RA Dr. Jan Biermann, LL.M.

(Cambridge), Prof. Dr. Jens-Hinrich Binder, LL.M. (London), RA Dr. Patrick Cichy, MBA (Wales), RA StB Dr. Sascha Engelbach, Prof. Dr. Philipp Gann, RA Falko Glasow, RA Dr. Alexander Glos,

Dr. André Hater, RAin Dr. Janina Heinz, Dr. Peter Henseler,

Prof. Dr. Jan-Philipp Hoffmann, Adrian Kämmler, Dr. Andreas Keese, Dr. Anne Kleppe, RA Dr. Kai Krieger, Dipl.-Kffr. Maike Kronbichler,

RA Dr. Klaus Lackhoff, LL.M. (Iowa), Steffen Laufenberg,

RA Dr. Nicholas Lütgerath, RA Dr. Heinrich Nemeczek, LL.M. (Harvard),

Prof. Dr. Werner Neus, Dr. Elma Sefer Periškić, RA Sebastian Pitz,

Dr. Inka Puppe, Prof. Dr. Jan Riepe, RA Andreas Steck, RA Jan Struckmann,

RA Dr. Thomas Voland, LL.M. (Nottingham), RA Dr. Benedikt Wolfers, M.A.

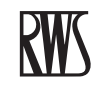

RWS Verlag Kommunikationsforum GmbH \& Co. KG · Köln 
Die Deutsche Nationalbibliothek verzeichnet diese Publikation in der Deutschen Nationalbibliografie; detaillierte bibliografische Daten sind im Internet über http://dnb.d-nb.de abrufbar.

(C) 2020 RWS Verlag Kommunikationsforum GmbH \& Co. KG

Postfach 2701 25, 50508 Köln

E-Mail: info@rws-verlag.de, Internet: http://www.rws-verlag.de

Das vorliegende Werk ist in all seinen Teilen urheberrechtlich geschützt. Alle Rechte vorbehalten, insbesondere das Recht der Übersetzung, des Vortrags, der Reproduktion, der Vervielfältigung auf fotomechanischem oder anderen Wegen und der Speicherung in elektronischen Medien.

Satz und Datenverarbeitung: SEUME Publishing Services GmbH, Erfurt Druck und Verarbeitung: CPI books GmbH, Leck 